\title{
The influence of quantum well and barrier thicknesses on photoluminescence spectra of InGaAs/AlInAs superlattices grown by LP-MOVPE
}

\author{
Adriana ŁOZIŃSKA*, MiKoŁaJ BADURA, Katarzyna BIELAK, \\ BEATA ŚCIANA, MAREK TŁACZAŁA
}

Faculty of Microsystem Electronics and Photonics, Wrocław University of Science and Technology, Janiszewskiego 11/17, 50-372 Wrocław, Poland

${ }^{*}$ Corresponding author: adriana.lozinska@pwr.edu.pl

\begin{abstract}
In the presented work, the influence of the quantum well and barrier thicknesses on optical characteristics of InGaAs/AlInAs superlattices was reported. Six different structures of $\operatorname{In}_{0.53} \mathrm{Ga}_{0.47} \mathrm{As} /$ $\mathrm{Al}_{0.48} \mathrm{In}_{0.52} \mathrm{As}$ superlattices lattice-matched to InP were grown by low pressure metal organic vapour phase epitaxy (LP-MOVPE). Optical properties of the obtained structures were examined by means of photoluminescence spectroscopy. This technique allows quick, simple and non-destructive measurements of radiative optical transitions in different semiconductor heterostructures. The analysis of recorded photoluminescence spectra revealed the influence of the quantum well and barrier thicknesses on the emission line energy.
\end{abstract}

Keywords: photoluminescence, quantum cascade lasers, MOVPE.

\section{Introduction}

A superlattice is a periodic structure of layers of two or more different materials. It is important to realize a difference between superlattices and multiple quantum wells, because they are very comparable in their structure, except that unlike the multiple quantum wells, the superlattice barrier thickness is small enough that electron states interpenetrate between individual quantum wells [1]. Superlattices based on InGaAs/AlInAs heterostructure are mostly used in the construction of quantum cascade lasers (QCLs) [르, $\underline{3}]$. The emission wavelength of QCLs based on InGaAs/AlInAs lattice-matched to InP substrate, covers the infrared region from 3.5 to $24 \mu \mathrm{m}$, which gives the opportunity to apply them in applications in chemical and biological sensing or spectroscopy [4]. The QCL core contains hundreds or even thousands of repetitions of thin layers with a thickness in the range of $0.5-10 \mathrm{~nm}$. In the case of such sophisticated structures, molecular beam epitaxy (MBE) is usually used, because it guarantees a specific composition in individual 
layers and very high quality of interfaces between them. Therefore, the epitaxial growth of such layers by metal organic vapour phase epitaxy (MOVPE) technique is very challenging and it is important to find the optimal technological parameters. The growth kinetics depends on several factors such as: a flow rate of precursors, process pressure and temperature which strongly affect the growth of individual layers [ $\underline{5}$ ]. In the case of layers with a thickness of a few nanometers, many measurement methods cannot be used. Photoluminescence (PL) is one of the most useful techniques for optical characterization of different semiconductor structures dedicated to optoelectronic devices, especially emitters [6]. PL spectra allow to determine the band gap energy, the dependence of the peak location of the maximum emission on the temperature or the excitation power.

The paper presents optical characterization of InGaAs/AlInAs low-dimensional structures in which InGaAs is a quantum well (QW) and AlInAs is a barrier (B).

\section{Experimental details}

Six different structures of InGaAs/AlInAs superlattices were grown by low pressure MOVPE (LP-MOVPE). The designed material parameters of investigated structures are collected in Table 1.

$\mathrm{T}$ a b 1 e 1. List of project assumptions of the investigated InGaAs/AlInAs superlattices: number of repetitions, thickness of $\operatorname{In}_{0.53} \mathrm{Ga}_{0.47} \mathrm{As}$ quantum well $\left(d_{\mathrm{QW}}\right)$ and thickness of $\mathrm{Al}_{0.48} \mathrm{In}_{0.52} \mathrm{As}$ barrier $\left(d_{\mathrm{B}}\right)$.

\begin{tabular}{lllc}
\hline Sample & $\begin{array}{l}\text { Number } \\
\text { of repetitions }\end{array}$ & $d_{\mathrm{QW}}[\mathrm{nm}]$ & $d_{\mathrm{B}}[\mathrm{nm}]$ \\
\hline A & 50 & 10 & 1 \\
B & 50 & 10 & 3 \\
C & 50 & 10 & 5 \\
D & 50 & 10 & 10 \\
E & 50 & 5 & 10 \\
F & 50 & 2 & 10 \\
\hline
\end{tabular}

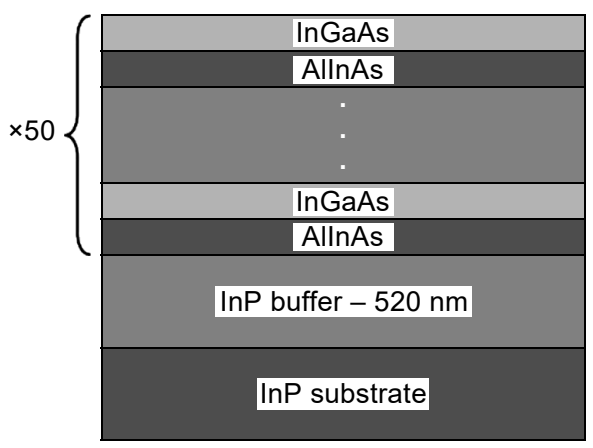

Fig. 1. A sectional view of the investigated InGaAs/AlInAs/InP heterostructures. 
Each sample consisted of 50 repetitions of two layers: the InGaAs quantum well and the AlInAs barrier lattice-matched to InP (Fig. 1). In the case of the samples A-D, the thickness of InGaAs quantum well $d_{\mathrm{QW}}$ was constant of $10 \mathrm{~nm}$ while the thickness of AlInAs barrier was changed 1, 3, 5 to $10 \mathrm{~nm}$. For the samples D, E and F, the thickness of AlInAs barrier $d_{\mathrm{B}}$ was kept constant of $10 \mathrm{~nm}$ while the thickness of InGaAs quantum well $d_{\mathrm{QW}}$ was varied from 2,5 to $10 \mathrm{~nm}$.

The epitaxial processes were carried out using AIXTRON $3 \times 2^{\prime \prime}$ FT CCS system at the pressure of $100 \mathrm{mbar}$. The growth temperature was $645^{\circ} \mathrm{C}$. A high purity hydrogen was applied as a carrier gas while trimethylaluminium (TMAl), trimethylgallium (TMGa), trimethylindium (TMIn), arsine $\left(\mathrm{AsH}_{3}\right)$ and phosphine $\left(\mathrm{PH}_{3}\right)$ were used as III- and V-group element sources, respectively. The superlattice composition was controlled by the hydrogen flow rate through TMGa and TMAl saturators at the fixed hydrogen flow rate through TMIn saturator. The optical properties of the fabricated samples were measured by means of low temperature photoluminescence (LT PL).

\section{Results}

PL spectra were measured at low temperature $(88 \mathrm{~K})$ using AlGaAs/GaAs laser excitation of $641 \mathrm{~nm}$ (continuous wave $\mathrm{CW}$, optical power $10 \mathrm{~mW}$ ). As a detector, the StellarNet DW ARF-STAR NIR-100 spectrometer was employed (InGaAs photodiode, $600 \mathrm{~g} / \mathrm{mm}$, integration time $100 \mathrm{~ms}, 0.5 \mathrm{~nm}$ resolution).

In the case of quantum cascade lasers, one of the most important properties is a sharp and narrow peak of the maximum emission. According to the theory with decreasing the thickness of the quantum well, the peak should shift towards shorter wavelengths (blue shift) []].

$\mathrm{SL} 50 \times \operatorname{lnGaAs}\left(\mathrm{d}_{\mathrm{QW}}\right) /$ AllnAs (barrier) $10 \mathrm{~nm} / \mathrm{lnP}$ buf $520 \mathrm{~nm} / \mathrm{lnP}: \mathrm{S}$

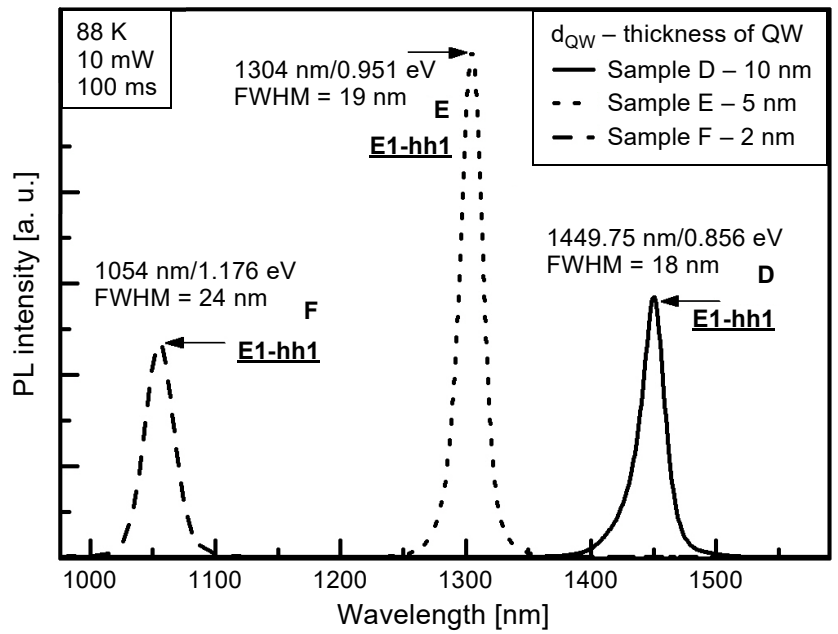

Fig. 2. PL spectra of the samples D, E, F recorded at $88 \mathrm{~K}$ with the excitation power of $10 \mathrm{~mW}$. 
PL spectra of three samples D, E and $\mathrm{F}$ in which the variable parameter was quantum well thickness $d_{\mathrm{QW}}(10,5$ and $2 \mathrm{~nm}$, respectively) are shown in Fig. 2. In those samples optical transitions come from the electron and heavy hole on the first level (E1-hh1). In the case of the sample E, the PL peak location was shifted toward shorter wavelengths of $150 \mathrm{~nm}$ in comparison to the sample $\mathrm{D}$, in which the quantum well thickness was twice larger. The separation between PL peaks of the samples E and F was $250 \mathrm{~nm}$, what corresponds to the reduction in the quantum well thickness 2.5 times. The observed changes of the PL peak position for different InGaAs quantum well thicknesses confirm the theory $[\underline{1}, 7]$. No additional optical transitions between light or heavy holes and electrons were observed in the presented PL spectra, what can result from a high quality of the measured heterostructures.

PL spectra of the samples A, B, C and D, in which the variable parameter was barrier thickness $d_{\mathrm{B}}(1,3,5$ and $10 \mathrm{~nm}$, respectively) are shown in Fig. 3. In those samples, optical transitions associated with electron and heavy hole on the first level (E1-hh1) are observed. In the case of the samples B, C and D, the position of maximum emission does not change distinctly (only a few nanometers). The shift of the maximum emission by $2 \mathrm{~nm}(\sim 1 \mathrm{meV})$ in the case of the sample B was probably caused by a change of the InGaAs quantum well thickness $d_{\mathrm{Qw}}$ by half of a lattice parameter. The small influence of the barrier thickness on the PL line energy indicates that the emission depends mainly on the quantum well thickness, which was constant for the considered samples. The situation differs when the barrier thickness is very narrow of $1 \mathrm{~nm}$ (sample A). In the case of this sample, the position of maximum emission shifts towards the longer wavelengths of $30 \mathrm{~nm}$ and a broadening of PL spectrum is observed (FWHM $=41 \mathrm{~nm})$. This is caused by the formation of a miniband characteristic for superlattice structure [1].

$\mathrm{SL} 50 \times \operatorname{lnGaAs}(\mathrm{QW}) 10 \mathrm{~nm} / \mathrm{AllnAs}\left(\mathrm{d}_{\mathrm{B}}\right) / \operatorname{lnP}$ buf $520 \mathrm{~nm} / \mathrm{lnP}: \mathrm{S}$

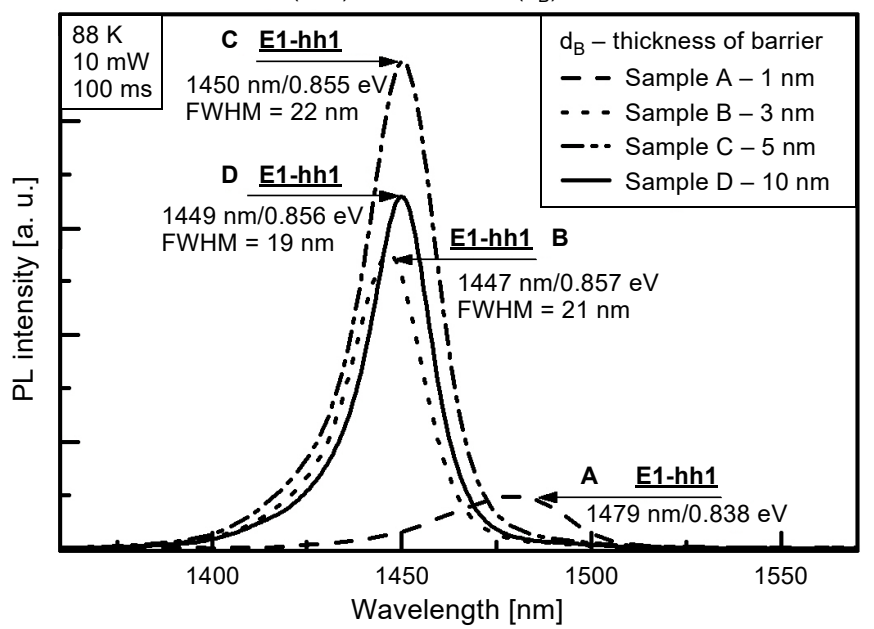

Fig. 3. PL spectra of the samples A, B, C and D recorded at $88 \mathrm{~K}$ with the excitation power of $10 \mathrm{~mW}$. 
$\mathrm{T}$ a b 1 e 2. Transitions observed on PL spectra and calculated optical transitions between electrons and light or heavy holes.

\begin{tabular}{lccccc}
\hline Sample & $d_{\mathrm{QW}}[\mathrm{nm}]$ & $d_{\mathrm{B}}[\mathrm{nm}]$ & $\begin{array}{l}\text { Transition on } \\
\text { PL spectra }[\mathrm{meV}]\end{array}$ & \multicolumn{2}{c}{ Calculated transition [meV] } \\
\cline { 5 - 6 } & 10 & 1 & 838 & E1-hh1 & E1-lh1 \\
B & 10 & 3 & 857 & 844 & 858 \\
C & 10 & 5 & 855 & 856 & 880 \\
D & 10 & 10 & 856 & 856 & 879 \\
E & 5 & 10 & 951 & 856 & 879 \\
F & 2 & 10 & 1176 & 944 & 993 \\
\hline
\end{tabular}

In order to confirm the results of the PL experiment, the theoretical verification was calculated on the basis of the theory contained in the articles [ $\underline{8}, \underline{9}]$. Energies of quantum wells transitions are determined by the approximation of effective mass calculated according to Pikus-Bir Hamiltonian (Table 2). To determine electrons and holes levels in the structures discussed in this article, one band $\mathbf{k} \cdot \mathbf{p}$ model has been applied [].

Based on the results of the calculations contained in Table 2, compliance with the results obtained from the photoluminescence measurement was found. Slight differences in the obtained results may be due to the discrepancy between the received and the proposed composition or the thickness of the layers.

\section{Conclusions}

Six InGaAs/AlInAs structures were grown on InP substrate by LP-MOVPE. The influence of the quantum well and barrier thicknesses on PL spectra was described. A shift of the emission line towards the shorter wavelengths caused by decreasing of the quantum well thickness was observed. The analysis of PL spectra recorded for the structures with different barrier thickness revealed that only the sample A with the narrowest barrier of $1 \mathrm{~nm}$ exhibits features attributed to a superlattice structure. Based on theoretical calculations, compliance with the results of the PL spectra was found. Small discrepancies in the results may indicate a change in the composition or thickness of individual layers, which is why it is worth comparing optical measurement methods with others, e.g. X-ray diffraction. The obtained results prove that photoluminescence can be used as a measurement technique even in such sophisticated structures. The next stage of research will be focused on determination of the maximum barrier thickness allowing the formation of the superlattice miniband.

Acknowledgements - This work was co-financed by Wrocław University of Science and Technology statutory grants and by the Polish National Centre for Research and Development through projects PBS2/ A3/15/2013 (PROFIT), TECHMAT-STRATEG1/347510/15/NCBR/2018 (SENSE). 


\section{References}

[1] Kasap S., Capper P., Springer Handbook of Electronic and Photonic Materials, 2nd Ed., Springer International Publishing AG, 2017, pp. 1037-1055.

[2] Bugajski M., Gutowski P., Karbownik P., Kolek A., Haldaś G., Pierściński K., Pierścińska D., Kubacka-Traczyk J., Sankowska I., Trajnerowicz A., Kosiel K., Szerling A., Grzonka J., KurzydŁowski K., Slight T., Meredith W., Mid-IR quantum cascade lasers: device technology and non-equilibrium Green's function modelling of electro-optical characteristics, Physica Status Solidi (B) 251(6), 2014, pp. 1144-1157, DOI: 10.1002/pssb.201350322.

[3] Faist J., Capasso F., Sivco D.L., Hutchinson A.L., Chu S.G., Cho A.Y., Short wavelength ( $\lambda$ 3.4 $\mu \mathrm{m})$ quantum cascade laser based on strained compensated InGaAs/AlInAs, Applied Physics Letters 72(6), 1998, pp. 680-684, DOI: 10.1063/1.120843.

[4] Huang Y., Ryou J.H., Dupuis R.D., Pflügl C., Capasso F., Sun K., Fischer A.M., Ponce F., Optimization of growth conditions for In GaAs/InAlAs/InP quantum cascade lasers by metalorganic chemical vapor deposition, Journal of Crystal Growth 316(1), 2011, pp. 75-80, DOI: 10.1016/j.jcrysgro. 2010. $\underline{12.028}$.

[5] Badura M., Bielak K., Ściana B., Radziewicz D., Pucicki D., Dawidowski W., Żelazna K., KudRAWIEC R., TŁaCZAŁA M., Technology and properties of low-pressure metalorganic vapour phase epitaxy grown InGaAs/AlInAs superlattice for quantum cascade laser applications, Optica Applicata 46(2), 2016, pp. 241-248, DOI: 10.5277/oa160208.

[6] Wójcik-Jedlińska A., Wasiak M., Kosiel K., Bugajski M., Photoluminescence characterization of AlGaAs/GaAs test superlattices used for optimization of quantum cascade laser technology, Optica Applicata 39(4), 2009, pp. 967-974.

[7] Wang Y., Sheng X., Guo Q., Li X., Wang S., Fu G., Mazur Y.I., Maidaniuk Y., Ware M.E., Salamo G.J., Liang B., Huffaker D.L., Photoluminescence study of the interface fluctuation effect for InGaAs/InAlAs/InP single quantum well with different thickness, Nanoscale Research Letters 12(1), 2017, article 229, DOI: 10.1186/s11671-017-1998-8.

[8] Kudrawiec R., Kopaczek J., Polak M.P., Scharoch P., Gladysiewicz M., Misiewicz J., Richards R.D., BAStiman F., DAVID J.P.R., Experimental and theoretical studies of band gap alignment in $\mathrm{GaAs}_{1-x} B i_{x} /$ GaAs quantum wells, Journal of Applied Physics 116(23), 2014, article 233508, DOI: $10.1063 / 1.4904740$.

[9] Vurgaftman I., Meyer J.R., Ram-Mohan L.R., Band parameters for III-V compound semiconductors and their alloys, Journal of Applied Physics 89(11), 2001, pp. 5815-5875, DOI: 10.1063/1.1368156. 